\title{
Polyamidoamine nanoparticles as nanocarriers for the drug delivery to malaria parasite stages in the mosquito vector
}

\author{
Patricia Urbán $^{1}$, Elisabetta Ranucci ${ }^{2}$, and Xavier Fernàndez-Busquets ${ }^{3,4,5}$ \\ ${ }^{1}$ European Commission, Joint Research Centre, Institute for Health and Consumer Protection, via E. \\ Fermi 2749, IT-21027, Ispra, Varese, Italy \\ ${ }^{2}$ Dipartimento di Chimica, Università degli Studi di Milano, via Golgi 19, IT-20133 Milano, Italy \\ ${ }^{3}$ Nanomalaria Group, Institute for Bioengineering of Catalonia (IBEC), Baldiri Reixac 10-12, ES- \\ 08028 Barcelona, Spain \\ ${ }^{4}$ Barcelona Institute for Global Health (ISGlobal, Hospital Clínic-Universitat de Barcelona), \\ Rosselló 149-153, ES-08036 Barcelona, Spain \\ ${ }^{5}$ Nanoscience and Nanotechnology Institute (IN2UB), University of Barcelona, Martí i Franquès 1, \\ ES-08028 Barcelona, Spain
}

\begin{abstract}
Summary
Malaria is arguably one of the main medical concerns worldwide because of the numbers of people affected, the severity of the disease and the complexity of the life cycle of its causative agent, the protist Plasmodium spp. With the advent of nanoscience, renewed hopes have appeared of finally obtaining the long sought-after magic bullet against malaria in the form of a nanovector for the targeted delivery of antimalarial compounds exclusively to Plasmodium-infected cells, thus increasing drug efficacy and minimizing the induction of resistance to newly developed therapeutic agents. Poly(amidoamine) (PAA)-derived nanovectors combine into a single chemical structure drug encapsulating capacity, antimalarial activity, low unspecific toxicity, specific targeting to Plasmodium, optimal in vivo activity, and affordable synthesis cost. After having shown their efficacy in targeting drugs to intraerythrocytic parasites, now PAAs face the challenge of spearheading a new generation of nanocarriers aiming at the malaria parasite stages in the mosquito vector.
\end{abstract}

KEYWORDS: Anopheles; antimalarial drugs; malaria; mosquitoes; nanomedicine; nanotechnology; Plasmodium; polymers; poly(amidoamine)s; targeted drug delivery

\section{Malaria: a main health concern with an economic bias}

Progress in shrinking the geographical range of endemic malaria has been remarkable, and since the launching of the World Health Organization (WHO)-led Global Malaria Eradication Campaign in 1955, 79 countries have eliminated malaria and the proportion of the world's population living in endemic regions has decreased more than 50\% [1]. Fifty years ago, malaria had been eliminated from many areas of the world through a combination of drug treatments and vector control interventions [2]. However, efforts were gradually abandoned from 1969 to 1976 due to the realization that the objective of eradication was unlikely to be easily achieved: the imperviousness of the vector to insecticides and the evolution of drug-resistant parasite strains severely impaired the WHO program [3]. In the 1990s control strategies were accelerated [4] through the creation of several research and public health coalitions, such as the Multilateral Initiative on Malaria, the Global Fund to Fight AIDS, Tuberculosis and Malaria, the U.S. President's Malaria Initiative and the Roll Back Malaria Partnership.

Increased prevention and control measures have led to a reduction in malaria mortality rates by more than $42 \%$ globally since 2000 . However, an estimated 3.3 billion people are at risk of being infected and developing symptoms, and 1.2 billion are at high risk ( $>1$ in 1000 chance of getting malaria in a year) [5], particularly in Africa, where the annual economic burden of the disease has been calculated to be around 12 billion US\$ in direct costs and to reduce GDP growth by $1.3 \%$ [6]. According to recent estimates, 198 million cases of malaria occurred worldwide in 2013 
(uncertainty range 124-283 million) and the disease led to 584,000 deaths (uncertainty range 367,000-755,000), but an independent study suggests that mortality could be twice as much if untreated and undiagnosed cases are included [7]. People living in the poorest countries are the most vulnerable, with approximately $90 \%$ of deaths in Africa, of which $78 \%$ were children under 5 years of age $[8,9]$. International and domestic funding for malaria control and elimination totaled US\$ 2.7 billion in 2013 [5]. Although this represented a threefold increase since 2005, it is still significantly below the estimated US\$ 5.1 billion that is required to achieve global targets for control and elimination. Total malaria funding will only match resource needs if international and domestic funders prioritize further investments for malaria control [5]. The current trend of global warming and generalized transcontinental travel, added to the growing number of displaced populations in endemic areas due to political and economic reasons, threatens with expanding the disease range. Malaria eradication is now on the global research agenda [10], but current vaccines in clinical assays do not offer prospects of complete protection [11] and the available drugs are rapidly losing efficacy. Thus, there is an urgent need to invest in the development of new medicines and therapeutic strategies $[12,13]$ working through radically new mechanisms. These new approaches should ideally: (i) address drug-resistance issues, (ii) have a rapid onset of action, (iii) be safe, and (iv) cure malaria in a single dose.

\section{Pathophysiology of malaria}

Five Plasmodium species cause disease in humans, namely $P$. vivax, $P$. ovale, $P$. malariae, $P$. knowlesi $[14]$ and $P$. falciparum, with the latter being responsible for the most deadly and severe cases. When taking a blood meal, the female Anopheles mosquito inoculates Plasmodium sporozoites (Figure 1) that in the liver infect hepatocytes and proliferate into thousands of merozoites [15]. Merozoites invade red blood cells (RBCs), and replicate asexually through ring, trophozoite and schizont stages to produce daughter cells that invade new RBCs to perpetuate the blood-stage cycle. Some parasites eventually differentiate into sexual stages, female or male gametocytes that are ingested by a mosquito from peripheral blood. Following fertilization in the insect's midgut, the zygote differentiates into an ookinete that moves through the midgut epithelium and forms an oocyst, which releases sporozoites. The malaria transmission cycle is restarted when sporozoites migrate to the salivary glands and are injected into a human with the mosquito's next bite. Because the blood-stage infection is responsible for all symptoms and pathologies of malaria, Plasmodium-infected RBCs (pRBCs) are a main chemotherapeutic target [16]. Since antimalarial drug delivery currently relies on compounds with little or no specificity for pRBCs, the administration of most drugs requires high doses. However, such unspecificity often demands a low upper concentration threshold to minimize undesirable side-effects in non-target cells, thus incurring the risk of sublethal doses favoring the evolution of resistant pathogen strains [17]. 


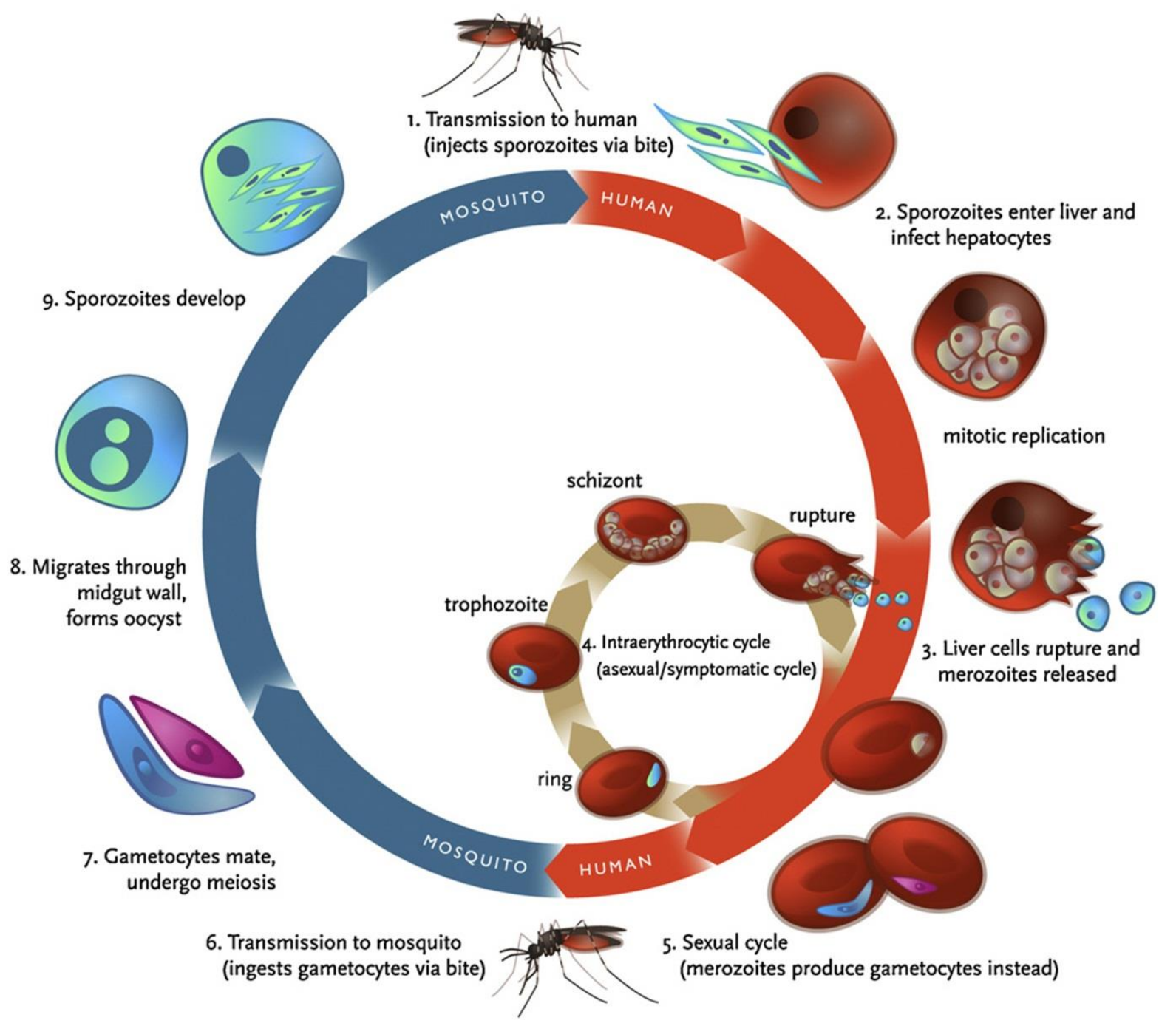

Figure 1. Life cycle of the malaria parasite. Transmission of malaria occurs through a vector, the mosquito, which ingests gametocytes - the sexual form of the parasite- when feeding on an infected human. Male and female gametocytes mate in the mosquito gut, undergo meiosis, and then migrate through the midgut wall to form an oocyst, within which thousands of sporozoites develop. These are then injected into a human during the next blood meal(s), where they rapidly make their way to the liver, infect hepatocytes and begin asexually (mitotically) replicating. After a period of ca. 6-15 days the liver schizonts rupture, releasing thousands of merozoites into the blood where they invade red blood cells, inside which the parasite progresses through a set of stages (ring, trophozoite and schizont) and produces an average of 16 new daughter merozoites per schizont. The schizonts then burst in near synchrony with other parasites, producing the characteristic fever cycle that embodies the clinical manifestations of the disease. With each replication, some of the merozoites develop into gametocytes, which can then infect susceptible mosquitoes, bringing the transmission cycle full circle. From [18], with permission.

\section{Nanotechnology against malaria}

Because malaria pathophysiology is so complex and the disease is so widespread, it is generally accepted that to achieve eradication a combination of weapons will be needed [19]. These include the improvement of existing approaches and the development of new ones [20], with drug therapy remaining the mainstay of treatment and prevention [21], and nanotechnology being able to provide innovative useful tools [22]. The objective of delivering drugs exclusively to a selected target site with minimal exposure for adjacent healthy cells or tissues is the holy grail of the fast-developing nanomedicine field [23]. Encapsulation of drugs in targeted nanovectors is a rapidly growing area with a clear applicability to infectious disease treatment [24], and pharmaceutical nanotechnology 
has been identified as a potentially essential tool in the future fight against malaria $[25,26]$.

Nanomedicine, which uses nanosized tools for treatment of disease [27], can fulfill the objective of achieving the intake of total amounts sufficiently low to be innocuous for the patient, but locally still lethal for the parasite. Mainly because of the lack of economic incentives, the application of nanotechnology to malaria has been traditionally neglected: a search in the Web of Science for the terms "nanomedicine" and "malaria" yields only ca. 30 peer-reviewed publications. The reasons for this gap in nanomedical research are surely varied, but among them are the lack of interest of a profit-seeking industry and the timid support of public administrations to small groups working off the main path of developed world diseases. Actually, the implementation of novel delivery approaches is less expensive than finding new antimalarial drugs and may optimize their rate of release [28]. Current immunoliposomal prototypes engineered for the delivery of antimalarial drugs specifically to pRBCs [29,30] rely on antibody targeting and contain special lipids, making their synthesis too expensive for practical widespread use in the routine treatment of most malaria cases, which are in regions with limited economic resources. An essential aspect for the successful development of antimalarial nanomedicines resides on the choice of encapsulating and targeting elements, of which it has to be considered their biocompatibility, cell specificity, binding affinity, ease of modification and conjugation to the drugs, production cost, scalability, amenability to oral administration formulation, and stability in mass production. Polymers offer virtually unlimited diversity in chemistry, dimensions and topology, rendering them a class of materials that is particularly suitable for applications in nanoscale drug delivery strategies [31].

\section{Poly(amidoamine)s}

Poly(amidoamines)s (PAAs) are a family of biodegradable and biocompatible polymers whose synthesis was reported more than 40 years ago [32] and since then they have been used in different fields [33], among which biomedical applications are prominent [34]. The preparation process of PAAs is simple, environmentally friendly and easily scalable, thus being suitable to be commercialized in regions characterized by low per capita income. PAAs can be synthesized by Michael type polyaddition of primary or bis-secondary amines to bis(acrylamide)s (Figure 2). The structures obtained present tert-amino and amido groups regularly arranged along the main chain, being in the absence of additional acid or basic substituents low to medium strength polymeric bases that can be classified as polyelectrolytes. PAAs are per se highly functionalized, but are also amenable to further chemical modification for special applications. Groups capable of reacting with activated double bonds under the conditions of PAA synthesis $\left(\mathrm{SH}, \mathrm{NH}_{2}, \mathrm{NR}\right.$ and $\mathrm{PH}_{2}$ ) cannot be introduced directly but instead they can be obtained by functionalization of purposely pre-synthesized polymers. Amphoteric PAAs derive from aminoacids or from carboxylated bis-acrylamides and carry both carboxyl and amino groups attached to the same monomer, and therefore in solution they change their net average charge as a function of $\mathrm{pH}$. By appropriately choosing the starting monomers, acidic and basic strengths of amino and carboxyl groups can be controlled in a way that the polymer switches from a prevailingly anionic to a prevailingly cationic state as a consequence of relatively modest $\mathrm{pH}$ changes [33].

a)

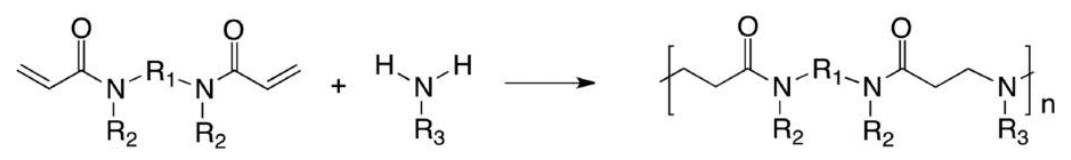

b)

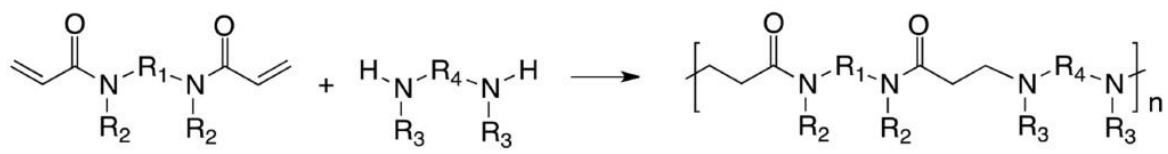

Figure 2. Synthesis of linear PAAs. R1, R2, R3 and R4 can be any alkyl residue eventually containing carboxyl, amide, ester or ether groups. 
The PAA polymerization reaction takes place in solvents carrying mobile protons, like water or alcohols, without the need for catalysts at room temperature [35,36]. High temperatures accelerate the polyaddition reaction rate but the resulting polymers have a lower molecular mass because of increased hydrolysis. PAA number- and weight-average molecular masses usually range between 5,000-40,000 and 10,000-70,000 respectively, with a polydispersity index of 1.5-2 depending on the purification method used after synthesis. Narrow polydispersity fractions can be obtained by fractionation techniques, such as ultrafiltration, size exclusion chromatography or field-flow fractionation. Nonamphoteric PAAs are soluble in water, but also in chloroform, lower alcohols, dimethyl sulfoxide and other polar solvents; on the other hand, amphoteric PAAs dissolve only in water. PAAs exhibit relatively large hydrodynamic volumes in solution if compared with vinyl polymers of similar mass, indicating a tendency to assume extended chain conformations in solution [34]. By introducing multifunctional amines in the polymerization mixture crosslinked structures can be obtained, which usually absorb large amounts of water and form hydrogels in aqueous media [37]. These hydrogels have been proven to possess good mechanic properties, biocompatibility, biodegradability and ability to induce cell adhesion and proliferation [38]. In aqueous media PAAs degrade within days or weeks to oligomeric products $[39,40]$, in a process strongly influenced by the structure of amide and amine moieties that increases at basic $\mathrm{pH}$ and higher temperatures $\left(40-60{ }^{\circ} \mathrm{C}\right)$. The degradation mechanism seems to be purely hydrolytic and not affected by the presence of lysosomal enzymes at pH 5.5 [41]. The toxicity of PAAs and their low molecular mass degradation products is two or more orders of magnitude lower than that of other polycations such as poly-L-lysine, polyethyleneimine or PAMAM dendrimers [34]. Amphoteric PAAs that at $\mathrm{pH} 7.4$ are mostly negatively charged are found to be non toxic, whereas strongly basic polymers positively charged at the same $\mathrm{pH}$ display significant cytotoxicity [42]. All PAAs cause more hemolysis at $\mathrm{pH} 5.5$ than at $\mathrm{pH} 7.4$ because protonation of the polymer backbone in acidic conditions increases its capacity to interact with and desestabilize the anionic RBC membrane [43].

\section{PAA applications in drug delivery}

Whereas some PAAs are captured by the liver or the kidney rapidly after i.v. injection [42], others circulate in the bloodstream for an extended period [44], showing a tendency to localize in tumours due to the enhanced permeability effect. This long blood residence time is an important feature to consider when selecting candidates for the design of drug delivery systems, since an increased circulation will facilitate interactions of polymers with the target cell, which usually internalizes them via the endocytic pathway [45]. PAAs were first tested for their ability to form polyelectrolyte stable complexes with heparin, in order to neutralise its anticoagulant activity $[34,46]$, results later extended to PAA-crosslinked resins, which have also been assayed for metal ion complexation [47]. Depending on their polymer content and formulation, these resins were able to incorporate from 30 to $100 \% \mathrm{w} / \mathrm{w}$ of heparin, without affecting other blood parameters [48]. Early in the 1970s several PAAs were shown to display inherent antitumour activity, reducing the number and average weight of Lewis lung tumour metastases after i.v. administration in mice, with different grades of toxicity and activity, although none of them were active against the primary tumour [49]. More recently, PAAs have been adopted as carriers for anticancer drugs such as mytomycin C [50], platinates [51] and doxorubicin. Conjugation of doxorubicin to polymers improves drug solubility, increases its blood half-life, decreases its toxicity, and mediates more efficient tumour targeting [52]. As the drug is inactive in its conjugated form and can be selectively released at the tumour site, doxorubicin can act primarily against cancer cells with minimal damage to healthy tissue. Doxorubicin has also been coupled to polymers via an acid-sensitive linker, facilitating the release of the drug in a biologically active form in the endosomal compartment [53].

As a promising tool to treat a variery of diseases, PAAs have been used as carriers for RNA and DNA as transfection promoters [54-57]. In particular, PAA polymers synthesized from bisacrylamides and incorporating a carboxylic acid group have been reported to achieve transfection efficiencies comparable to polyethyleneimine, the gold standard for polymer-assisted gene transfection, but with a significantly lower cytotoxicity [56]. Different modifications of PAAs have been published, for 
instance incorporating repetitive disulfide links in the main chain [58], linear or branched architectures [59], intercalating quaternary nicotinamides [55] and boronic acid moieties [54] as side groups, in order to combine stability of the polyplexes, high transfection efficiencies and low unspecific cytotoxicity [60]. In vitro assays demonstrated the ability of the polymers to form stable polyplexes, to interact with cell membranes in a non-disruptive way, to protect DNA from enzymatic degradation in the biological environment and to promote stable gene transfection in living cells [61]. PAAs have been found to be particularly suitable for the intracellular delivery of peptides and proteins $[62,63]$, which can be inserted for targeting purposes either as pendants or as integral portions of the polymer chain. Different functionalized PAAs have been optimized to obtain a fast and efficient protein release with lysozyme as a model cationic protein [63] or with $\beta$-galactosidase, which was successfully taken up into cells, whereas the enzyme alone could not be internalized [64]. These polymer-protein complexes are stable in extracellular media but disintegrate into low molecular mass fragments once inside the cells. The (partial) release of proteins from the complexes is induced by charge reversal at endosomal $\mathrm{pH}$, leading to decreased protein-polymer interaction, and by polymer degradation due to intracellular reduction of polymer disulfide linkages. Endosomal escape of the nanovessel and its cargo will depend on the different functionalities present in the polymer [65], e.g. positively charged groups are expected to have increased interactions with the membrane of the organelle and promote endosomal disruption [58]. Other PAA derivatives, such as branched polymers with arginine, maintain the cell internalization capacity of the amino acid, but having increased biocompatibility than argininerich cell penetrating peptides [66]. PAAs have been used as carriers for the antiviral compound Acyclovir [67], where in vitro experiments showed that polymer conjugation of the drug increased its activity. More recent results $[68,69]$, indicate that agmatine-containing PAAs possess antiviral activity which is not a mere consequence of cytotoxicity.

\section{PAAs for the targeted delivery of antimalarial drugs}

The PAA structures AGMA1, ISA1 and ISA23 (Figure 3) have been explored for the encapsulation and targeted delivery of the antimalarial drugs chloroquine and primaquine [70].

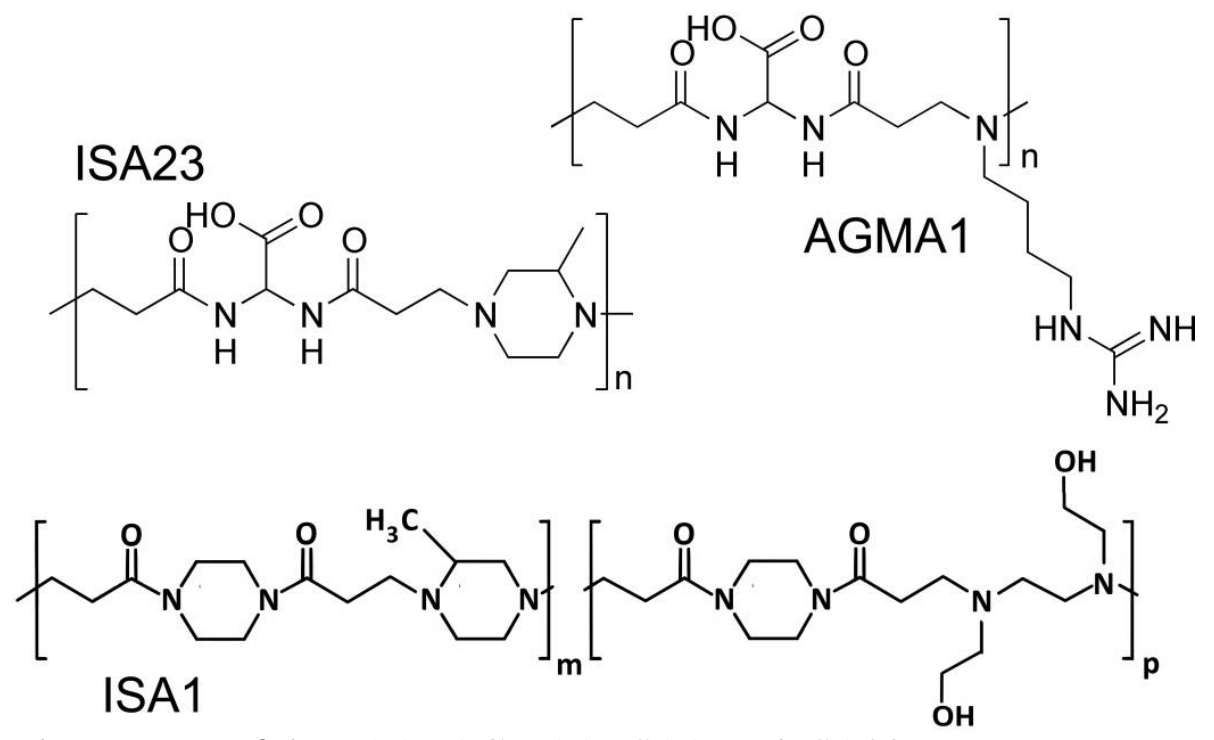

Figure 3. Chemical structures of the PAAs AGMA1, ISA1, and ISA23.

AGMA1 is obtained by polyaddition of 4-aminobutylguanidine (agmatine) with 2,2bis(acrylamido)acetic acid and contains tert-amine, carboxyl and guanidine groups. It is amphoteric with isoelectric point 10.0 and therefore at $\mathrm{pH} 7.4$ is prevailingly cationic with, on average, 0.55 excess positive charges per unit. ISA23 is obtained by polyaddition of 2-methylpiperazine with 2,2bis(acrylamido)acetic acid. Notwithstanding carrying two tert-amine groups and one carboxyl group per unit, it has isoelectric point 5.5, being prevailingly anionic at $\mathrm{pH} 7.4$ with, on average, 0.38 excess negative charges per unit. ISA1 is obtained by polyaddition of bis(acryloyl)piperazine 
with 2-methylpiperazine and bis(hydroxyethylethylenediamine). It is a rather weak polymeric base with, on average, 0.55 positive charges per unit at $\mathrm{pH}$ 7.4. All these polymers had been reported as vectors for the intracellular delivery of nucleic acids [56,61,71], whereas ISA1 and ISA23 had been also studied for protein delivery $[45,62,71]$ and as anticancer drug carriers [51,53]. ISA23 in particular has been proven to be endowed with stealth-like properties without selectively concentrating in the liver [44], while a significant portion of AGMA1 did show hepatic localization after intravenous injection in mice [42]. In intracellular compartments where the $\mathrm{pH}$ decreases to 6.5 (endosomes) and then to 5.0 (lysosomes), PAAs become prevailingly cationic and display endosomolytic properties [72]. Fluorescence microscopy revealed colocalization of ISA1 and ISA23 with Lysotracker, a marker for lysosome and late endocytic structures, and ISA1 also colocalized with the Early Endosomal Antigen 1 that accumulates in early endocytic structures [45]. Atomic force microscopy images (Figure 4) revealed a globular conformation for AGMA1, ISA23 and ISA1 adsorbed on mica substrates, showing a homogenous polymer size distribution with a hydrodynamic radius between 6 and $7 \mathrm{~nm}$ according to size exclusion chromatography analysis [70].
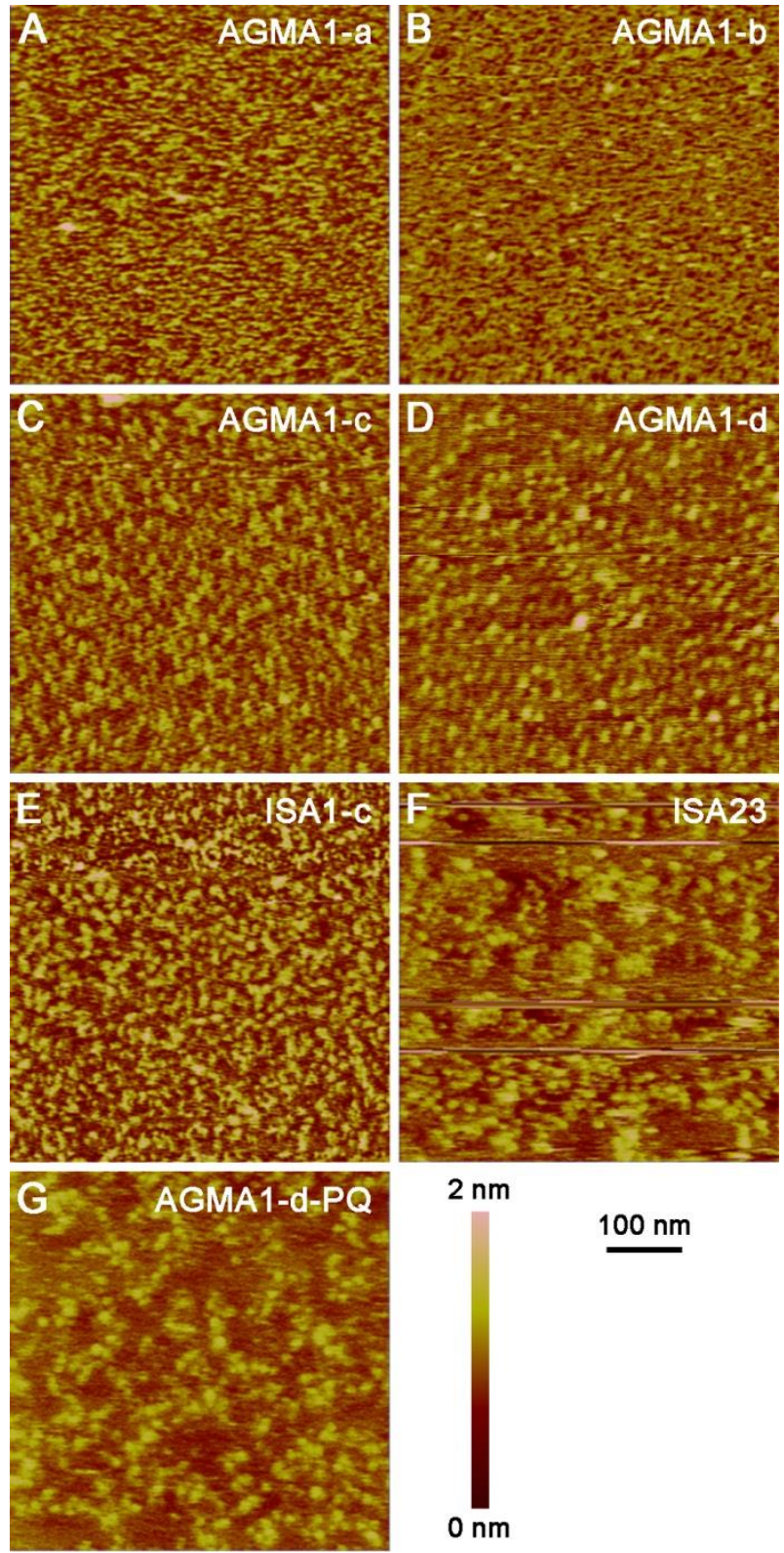

Figure 4. Atomic force microscope images in liquid of different fractions of AGMA1 (A-D) and ISA1 (E), of non-fractionated ISA23 (F), and of AGMA1 encapsulating the antimalarial drug primaquine $(\mathrm{G})$. From [70], with permission. 
pRBCs are known to be permeable to high molecular mass solutes up to diameters of $70 \mathrm{~nm}$ $[73,74]$, including peptides and proteins, with which PAAs share some features, such as the polyelectrolyte behavior and the presence of amide groups in the main chain. This led us to explore the potential of ISA1, ISA23 and AGMA1 as antimalarial drug carriers. Fluorescence-assisted cell sorting, confocal immunofluorescence and transmission electron microscopy results indicated that the tested polymers have specific targeting to pRBCs, and subcellular targeting to the parasite itself (Figure 5). In 4-day suppressive tests, mice infected with a lethal strain of the murine malaria species Plasmodium yoelii were freed of parasites and cured after intraperitoneal administration of chloroquine encapsulated in AGMA1 or ISA23 at a dose of $0.8 \mathrm{mg} \mathrm{kg}^{-1} \mathrm{day}^{-1}$, whereas the same amount of free drug was unable to cure the animals [70]. PAAs targeted different Plasmodium species and AGMA1 in particular possessed significant intrinsic antimalarial activity per se, showing binding to merozoites and probably inhibiting their invasion of new red blood cells. The ensuing prolonged exposure of the pathogens to the immune system might be applied to the design of new malaria vaccination approaches where PAAs could play a dual role as carriers of antimalarial drugs and as vaccination adjuvants. This unexpected synergistic effect combining therapeutics and prophylaxis represents a radically new approach to the treatment of malaria for which we propose the term theralaxis.
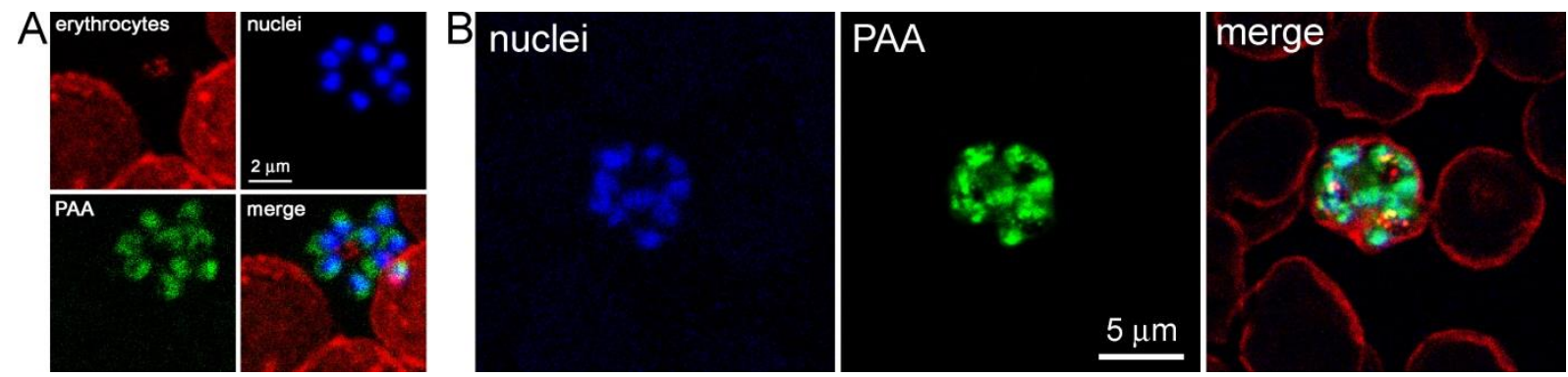

Figure 5. Confocal fluorescence microscopy targeting study of AGMA1 and ISA23 to $P$. falciparum and $P$. yoelii. FITC-labeled ISA23 (A) or AGMA1 (B) were added to either $P$. yoeliiinfected mouse blood, or to living P. falciparum cultures of the 3D7 strain, respectively. After 90 min of incubation the samples were processed for confocal fluorescence microscopy analysis. PAAFITC localization is shown in green, DAPI (blue) staining of Plasmodium nuclei was used to reveal the parasites, and the RBC plasma membrane is shown in red. From [70], with permission.

\section{Antimalarial drug delivery to mosquitoes}

Current chemotherapeutic approaches against malaria are targeted at the asexual, blood-stage parasites responsible for all symptoms and pathologies of the disease [16]. However, as in the bloodstream of a malaria patient there can be several hundred billion pRBCs, multiple-dose administrations of drugs are usually required to clear infections. This continuous exposure of Plasmodium to antimalarials increases the likelihood of resistance evolution, which will rapidly decrease drug efficacy. The threat of resistance-driven treatment failure is prompting research oriented to targeting the weakest forms of the pathogen represented by smaller populations, which are less likely to contain resistant individuals that would benefit from the removal of susceptible parasites [75]. The two main bottlenecks in the malaria cycle are found where the parasite is in one of the transmission stages that move between hosts [76]. A few thousand sporozoites can be packed inside the mosquito salivary glands, but only approximately 100 will be transferred to the human when Anopheles bites; this is several orders of magnitude fewer parasites than are found in an active blood stage infection. However, the short time that free sporozoites remain in the circulation is a serious obstacle to targeting them. A second bottleneck occurs during sexual development, when ca. $0.2-1 \%$ of the intraerythrocytic parasites may develop into gametocytes per round of schizogony. Although this still leaves an estimated $10^{8}-10^{9}$ parasites to be cleared from the blood circulation, 
targeting gametocytes can ease exposure of the pathogen to drugs and reduce the likelihood of resistance emerging [76].

However, a largely unexplored avenue in antimalarial drug development is targeting the parasite stages in the insect vector itself [77], an approach being barely investigated in the laboratory, and not implemented yet as part of a clinically feasible alternative therapy. Although the innate immune system of mosquitoes is capable of completely clearing a malaria infection [78], it is far from the sophisticated arsenal providing long-term protection in mammalian adaptive immunity. This might result in mosquito stages of Plasmodium having reduced defenses because they need to survive just a few days facing an immune surveillance not as demanding as in the human host. In addition, the richness of biological processes required for development in the mosquito likely withdraws from the parasite metabolic resources otherwise allocated to drug resistance. Drugs targeting early Anopheles stages must kill only ca. $5 \times 10^{3}$ parasites to free a mosquito from Plasmodium infection [79], and the absolute low corresponds to oocysts, of which there are only 25 in a single insect [76], being around for over a week. The recent appreciation that decreasing malaria prevalence requires strategies to reduce transmission through the mosquito [80] has prompted a renewed search for alternative therapeutic approaches. Unlike the asexual parasites, Plasmodium transmission forms are difficult and expensive to propagate and handle in the laboratory, but the first practical steps that shall eventually make possible these new objectives are being taken in the form of incipient protocols for the purification of $P$. falciparum ookinetes [81], although their culture has not been standardized $[82,83] . P$. vivax ookinetes can be grown in culture [84], but implementation of this assay would require both the stable transfection of the parasite, a process not yet achieved [85], and the routine availability of viable gametocytes en masse, currently not possible from culture. Gametocyte-to-ookinete development can be reproduced successfully in vitro for the rodent malaria parasite P. berghei [86]. However, these alternative cell targets incur the significant problem of their in vivo location in the mosquito.

Two approaches seem to offer some hope of being able to deliver transmission-blocking drugs to Anopheles, but it is difficult to say which one poses more daunting obstacles. Because gametogenesis, fertilization, and zygote differentiation into an ookinete occur in the mosquito within an environment essentially consisting of human blood, any compound affecting these processes can be delivered via the very blood meal of the insect. Thus, we can consider administering to humans antimalarials with a sufficiently long blood half-life to have good chances of being taken up by a mosquito during its bite. According to approximate estimates this might mean between 3 or 4 weeks in the blood of people living in endemic areas. In addition to patient compliance, we face the challenge of finding a drug escaping spleen and liver clearance and kidney filtration for a formidably long time span, and which in the end has to be present at therapeutic concentration in the infected insect. To the best of our knowledge, the only example of drug administration in this way to mosquitoes is the case of ivermectin [87], an insecticide which, at concentrations found in human blood after treatment, is toxic to all Anopheles species examined. However, the insect has to blood-feed on a treated subject (i.e. patient compliance is a parameter to consider), and resistances to ivermectin have been already documented [88].

\section{Direct antimalarial drug administration to mosquitoes}

The alternative to administering antimalarials to mosquitoes via human blood through the insect's blood meal is even more mind-boggling: delivering the drug directly to Anopheles. For this approach to work it is a must that the mosquito should have the need to ingest something between a first blood meal where gametocytes have been taken and the second infective bite once Plasmodium sporozoites have developed. There seems to be a lack of consensus as to whether during that time lapse Anopheles either rests in a secluded spot, or it does eventually drink some substance. But even if the mosquito is satiated for weeks following a blood meal, the very biology of the malaria parasite might assist us here. To keep blood fluid and prevent quick coagulation, Anopheles synthesizes an anti-hemostatic armamentarium containing, among others, the enzyme apyrase. Plasmodium inhibits apyrase [89] and in this way entices the mosquito to bite more because blood 
coagulates faster and Anopheles has to probe longer to get its full dinner, thereby increasing potentially infective host contacts. It can be expected then that infected insects will have a larger probability of probing a non-human source.

While female mosquitoes depend on protein-rich blood meals for egg maturation, both male and female mosquitoes are also attracted to and feed from plants. Plant nectar is an important, carbohydrate-rich nutrient source that provides essential energy for flight and, for some mosquito species, overwintering [90,91]. This phytoattraction has been successfully harnessed by malaria control efforts through attractive nectar baiting strategies, in which mosquitoes are lured to sugarwater blends spiked with plant volatiles and insecticides [92,93]. Suspected preferred host plants for Anopheles gambiae include Asteracaeae spp. and Ricinus communis [94]. Analysis of purified odorants from these plants has revealed enrichment of volatile compounds known as terpenes, including 10-carbon monoterpenes such as pinene and limonene, which at low concentrations have been shown to mediate attraction of Anopheles spp. [94,95]. Several approaches are currently available for oral delivery to mosquitoes by droplet or liquid feeding, through dry diets [96], or via nylon strips continuously dispensing synthetic mosquito attractants for several weeks [97,98]. Some of these methods have been already used to deliver to dipterans lipid-based [99] and chitosan nanoparticles [100]. Because Anopheles males can be easily fed from drug-containing sugar-baited traps, unlike females whose blood meal feeding habits complicate the design of a surrogate blood diet, it would be advisable to investigate the possible horizontal transfer of antimalarial drugs through sperm. Another interesting, though challenging, alternative to administering PAAencapsulated drugs to adult mosquitoes with the objective of eliminating Plasmodium from infected insects, is delivery to mosquito eggs. In such strategy, if PAA nanocarriers can be made to persist throughout metamorphosis, emerging mosquitoes might be endowed with prophylactic antimalarial capacity. A number of chemicals have been proposed as oviposition attractants for A. gambiae [101,102], with the sesquiterpene alcohol cedrol as one of the most promising candidates [103].

Highly concentrated drugs against Plasmodium gametes, ookinetes, oocysts or sporozoites could be directly dispensed to mosquitoes from fixed-volume containers where the drug does not become diluted with time as when it circulates in human blood. Such a strategy, because it is not designed for administration to humans, will bypass clinical trials that often delay for years the deployment of a new medicine, and will significantly reduce treatment development costs. Mosquito-dwelling transmission forms will likely be efficiently reached by specifically targeted nanovectors encapsulating the corresponding drugs, which in this way are protected from degradation before being ingested by the insect. A final bonus of delivering the nanocarriers directly to mosquitoes is that the usable nanoparticle size range becomes greatly expanded between a few nm and up to several microns for the direct delivery to females. Administration to insects will allow also for a not so strict vigilance on other nanocarrier characteristics such as zeta potential, toxicity of the chemical units constituting the nanovector, the nature, type and number of targeting units, or the nature, number, and amount of $\operatorname{drug}(\mathrm{s})$ loaded.

\section{Targeting mosquito stages of Plasmodium}

The three elements that constitute a targeted therapeutic nanovector (nanocapsule, targeting molecule and the drug itself) can be exchanged, as if they were LEGO parts, to obtain new structures better suited to each particular situation. Through modification of its constituting elements, nanovector design is susceptible of improvement and of adaptation to new targets such as different Plasmodium species or infected cells other than the erythrocyte. Of particular interest here, as discussed above, is the targeting of the transmission stages that allow transfer of the parasite between human and mosquito and vice-versa, which represent the weakest spots in the life cycle of the pathogen [77]. Heparin and heparan sulfate are targets for the circumsporozoite protein in the sporozoite attachment to hepatocytes during the primary stage of malaria infection in the liver [104]. Chondroitin sulfate proteoglycans in the mosquito midgut and synthetic chondroitin sulfate mimetics have been described to bind Plasmodium ookinetes as an essential step of host epithelial cell invasion [105,106], whereas ookinete-secreted proteins have been found to possess significant 
binding to heparin [107]. A synthetic polysulfonated polymer that mimics the structure of glycosaminoglycans present in the mosquito midgut surface has been used as a proof of concept for a transmission-blocking strategy [106]. The authors showed that the inhibition of Plasmodium development in the mosquito could be achieved by interfering in the interaction between the parasite and the mosquito midgut epithelium, which is a key step in the life cycle of the pathogen. This body of accumulated evidence suggests that glycosaminoglycans might be adequate to target antimalarial-loaded PAA-based nanovectors to Plasmodium mosquito stages, either through a direct entry into gametocytes, ookinetes, and sporozoites, or indirectly through delivery to pRBCs for those that will later differentiate into gametocytes.

Phenomenal experimental obstacles loom above this approach, which could only be pushed forward with a truly multidisciplinary research team involving chemists, physicians, entomologists, environmentalists, biochemists, evolutionary biologists, nanotechnologists, and many other professionals. Although eliminating a handful of oocysts in a bug seems easy enough, the sheer numbers of mosquitoes that have to be reached represents a challenge that will require a deep understanding of insect behavior and, likely, the development of new antimalarial drugs working through radically new mechanisms. But, if we take the pain of targeting the mosquito, wouldn't it be better just delivering insecticide? However, by wiping off an insect species we might be unbalancing the ecosystem in unpredictable ways, not to mention that besides Anopheles, the concocted broth can be a delicatessen for many other insects, some of them with known essential functions e.g. as pollinators, whose eradication might bring crop collapse and famine.

\section{Future perspective}

Future antimalarial strategies relying on drugs working through radically new mechanisms might demand direct delivery to Plasmodium stages in the mosquito of PAA-based targeted nanovectors loaded with these new medicines. The specifications to which the nanocarriers will likely have to fit are (i) a simple and scalable synthesis with affordable cost, (ii) the capacity to encapsulate a wide range of antimalarial drug structures, (iii) a long half-life of months without losing integrity before being ingested by the mosquito while preserving drug activity, (iv) an adequate degradation rate once inside female Anopheles to allow the drug entering Plasmodium, (v) a slower degradation rate once inside male Anopheles to allow the nanocarriers being horizontally transferred to females upon mating, (vi) a high solubility in mosquito artificial diets to allow for the maximum affordable concentrations, and (vii) a targeting as specific as possible to Plasmodium stages inside Anopheles (gametocytes, ookinetes, oocysts and sporozoites). As we have discussed above, PAA-based nanocarriers can fulfill these requirements and thus significantly contribute as a new weapon in a future scenario of malaria eradication.

\section{Executive summary}

\section{Malaria: a main health concern with an economic bias}

- There is an urgent need to invest in the development of new antimalarial medicines and therapeutic strategies working through radically new mechanisms.

\section{Pathophysiology of malaria}

- The unspecificity of toxic drugs demands low concentrations to minimize undesirable side-effects, thus incurring the risk of sublethal doses favoring the evolution of resistant Plasmodium strains.

\section{Nanotechnology against malaria}

- Drug therapy remains the mainstay of treatment and prevention against malaria, with nanotechnology being able to provide innovative useful tools.

- Pharmaceutical nanotechnology has been identified as a potentially essential tool in the future fight against malaria.

- Nanomedicine can fulfill the objective of achieving the intake of total amounts sufficiently low to be innocuous for the patient, but locally still lethal for the parasite.

- Mainly because of the lack of economic incentives, the application of nanotechnology to malaria has been traditionally neglected. 
- The development of novel delivery approaches is less expensive than finding new antimalarial drugs.

Poly(amidoamine)s

- The preparation process of PAAs is simple, environmentally friendly and easily scalable, thus being suitable to be commercialized in regions characterized by low per capita income.

PAA applications in drug delivery

- PAAs have been found to be particularly suitable for the intracellular delivery of peptides and proteins.

PAAs for the targeted delivery of antimalarial drugs

- PAAs have been shown to be targeted to Plasmodium.

Antimalarial drug delivery to mosquitoes

- A largely unexplored avenue in antimalarial drug development is targeting the parasite stages in the insect vector itself.

Direct antimalarial drug administration to mosquitoes

- Drug delivery to mosquitoes, because it is not designed for administration to humans, will bypass clinical trials that often delay for years the deployment of a new medicine.

Targeting mosquito stages of Plasmodium

- Glycosaminoglycans might be adequate to target antimalarial-loaded PAA-based nanovectors to Plasmodium mosquito stages.

\section{Acknowledgements}

This research was supported by grants 2013-0584 (Fondazione Cariplo, Italy), BIO2014-52872-R (Ministerio de Economía y Competitividad, Spain), which included FEDER funds, and 2014-SGR938 (Generalitat de Catalunya, Spain).

\section{References}

Papers of special note have been highlighted as:

* of interest; ** of considerable interest

1. Hay SI, Guerra CA, Tatem AJ, Noor AM, Snow RW. The global distribution and population at risk of malaria: past, present, and future. Lancet Infect. Dis. 4(6), 327-336 (2004).

2. Greenwood BM, Fidock DA, Kyle DE et al. Malaria: progress, perils, and prospects for eradication. J. Clin. Invest. 118(4), 1266-1276 (2008).

3. Nájera JA, González-Silva M, Alonso PL. Some lessons for the future from the Global Malaria Eradication Programme (1955-1969). PLoS Med. 8(1), e1000412-e1000412 (2011).

4. Mills A, Lubell Y, Hanson K. Malaria eradication: the economic, financial and institutional challenge. Malar. J. 7(Suppl 1), S11-S11 (2008).

5. World Health Organization. World Malaria Report 2014. http://www.who.int/malaria/publications/world_malaria_report_2014/report/en/ (2014).

** Updated overview of the progress in the fight against malaria and recommendations for its prevention and control.

6. Roll Back Malaria Partnership 2015 Report (2015).

7. Biamonte MA, Wanner J, Le Roch KG. Recent advances in malaria drug discovery. Bioorg. Med. Chem. Lett. 23(10), 2829-2843 (2013).

8. Vangapandu S, Jain M, Kaur K, Patil P, Patel SR, Jain R. Recent advances in antimalarial drug development. Med. Res. Rev. 27(1), 65-107 (2007).

9. Okiro EA, Al Taiar A, Reyburn H, Idro R, Berkley JA, Snow RW. Age patterns of severe paediatric malaria and their relationship to Plasmodium falciparum transmission intensity. Malar. J. 8, 4- (2009).

10. Kappe SH, Vaughan AM, Boddey JA, Cowman AF. That was then but this is now: malaria research in the time of an eradication agenda. Science 328(5980), 862-866 (2010). 
11. Moorthy VS, Newman RD, Duclos P, Okwo-Bele JM, Smith PG. Assessment of the RTS,S/AS01 malaria vaccine. Lancet Infect. Dis. 13(4), 280-282 (2013).

12. Alonso PL, Tanner M. Public health challenges and prospects for malaria control and elimination. Nat. Med. 19(2), 150-155 (2013).

13. Wells TN, Alonso PL, Gutteridge WE. New medicines to improve control and contribute to the eradication of malaria. Nat. Rev. Drug Discov. 8(11), 879-891 (2009).

14. White NJ. Plasmodium knowlesi: the fifth human malaria parasite. Clin. Infect. Dis. 46(2), 172-173 (2008).

15. Tuteja R. Malaria - an overview. FEBS J. 274(18), 4670-4679 (2007).

* This review provides an authoritative concise overview of malaria pathophysiology.

16. Griffith KS, Lewis LS, Mali S, Parise ME. Treatment of malaria in the United States: a systematic review. JAMA 297(20), 2264-2277 (2007).

17. Baird JK. Effectiveness of antimalarial drugs. N. Engl. J. Med. 352(15), 1565-1577 (2005).

18. Klein EY. Antimalarial drug resistance: a review of the biology and strategies to delay emergence and spread. Int. J. Antimicrob. Agents 41(4), 311-317 (2013).

* This paper explains how resistance evolves and spreads, whose understanding is crucial for developing strategies to contain its emergence.

19. Feachem RG, Phillips AA, Targett GA, Snow RW. Call to action: priorities for malaria elimination. Lancet 376(9752), 1517-1521 (2010).

20. Alonso PL. Malaria: deploying a candidate vaccine (RTS,S/AS02A) for an old scourge of humankind. Int. Microbiol. 9(2), 83-93 (2006).

21. Daily JP. Antimalarial drug therapy: the role of parasite biology and drug resistance. J. Clin. Pharmacol. 46(12), 1487-1497 (2006).

22. European Science Fundation. ESF Forward Look on Nanomedicine 2005. http://www.nanopharmaceuticals.org/files/nanomedicine.pdf (2005).

23. Saltzman M, Desai T. Drug delivery in the BME curricula. Ann. Biomed. Eng. 34(2), 270275 (2006).

24. Urbán P, Valle-Delgado JJ, Moles E, Marques J, Díez C, Fernàndez-Busquets X. Nanotools for the delivery of antimicrobial peptides. Curr. Drug Targets 13(9), 1158-1172 (2012).

25. Kuntworbe N, Martini N, Shaw J, Al-Kassas R. Malaria intervention policies and pharmaceutical nanotechnology as a potential tool for malaria management. Drug Dev. Res. 73, 167-184 (2012).

26. Urbán P, Fernàndez-Busquets X. Nanomedicine against malaria. Curr. Med. Chem. 21(5), 605-629 (2014).

* Review of the application of different areas of nanotechnology for the diagnosis and treatment of malaria.

27. Duncan R, Gaspar R. Nanomedicine(s) under the microscope. Mol. Pharmaceutics 8(6), 2101-2141 (2011).

28. Murambiwa P, Masola B, Govender T, Mukaratirwa S, Musabayane CT. Anti-malarial drug formulations and novel delivery systems: a review. Acta Tropica 118(2), 71-79 (2011).

29. Urbán P, Estelrich J, Cortés A, Fernàndez-Busquets X. A nanovector with complete discrimination for targeted delivery to Plasmodium falciparum-infected versus non-infected red blood cells in vitro. J. Control. Release 151(2), 202-211 (2011).

30. Urbán P, Estelrich J, Adeva A, Cortés A, Fernàndez-Busquets X. Study of the efficacy of antimalarial drugs delivered inside targeted immunoliposomal nanovectors. Nanoscale Res. Lett. 6, 620- (2011).

31. Paleos CM, Tsiourvas D, Sideratou Z, Tziveleka LA. Drug delivery using multifunctional dendrimers and hyperbranched polymers. Expert Opin. Drug Deliv. 7(12), 1387-1398 (2010).

32. Danusso F, Ferruti P. Synthesis of tertiary amine polymers. Polymer 11(2), 88-113 (1970).

33. Ferruti P. Poly(amidoamine)s: past, present, and perspectives. J. Polym. Sci. Part A: Polym. Chem. 51(11), 2319-2353 (2013). 


\section{** This review article provides the state of the art of the chemistry and applications of PAAs in different fields.}

34. Ferruti P, Marchisio MA, Duncan R. Polyamidoamines: biomedical applications. Macromol. Rapid. Commun. 23, 332-355 (2002).

35. Manfredi A, Ranucci E, Suardi M, Ferruti P. Polymerization kinetics of poly(amidoamine)s in different solvents. J. Bioact. Compat. Polym. 22(2), 219-231 (2007).

36. Ferruti P, Marchisio MA, Barbucci R. Synthesis, physico-chemical properties and biomedical applications of poly(amidoamine)s. Polymer 26(9), 1336-1348 (1985).

37. Ferruti P, Bianchi S, Ranucci E, Chiellini F, Piras AM. Novel agmatine-containing poly(amidoamine) hydrogels as scaffolds for tissue engineering. Biomacromolecules 6(4), 2229-2235 (2005).

38. Mauro N, Manfredi A, Ranucci E et al. Degradable poly(amidoamine) hydrogels as scaffolds for in vitro culturing of peripheral nervous system cells. Macromol. Biosci. 13(3), 332-347 (2013).

39. Ferruti P, Ranucci E, Bignotti F, Sartore L, Bianciardi P, Marchisio MA. Degradation behaviour of ionic stepwise polyaddition polymers of medical interest. J. Biomater. Sci. Polym. Ed. 6(9), 833-844 (1995).

40. Bignotti F, Sozzani P, Ranucci E, Ferruti P. NMR studies, molecular characterization, and degradation behavior of poly(amido amine)s. 1. Poly(amido amine) deriving from the polyaddition of 2-methylpiperazine to 1,4-bis(acryloyl)piperazine. Macromolecules 27(24), 7171-7178 (1994).

41. Ranucci E, Spagnoli G, Ferruti P, Sgouras D, Duncan R. Poly(amidoamine)s with potential as drug carriers: degradation and cellular toxicity. J. Biomater. Sci. Polym. Ed. 2(4), 303-315 (1991).

42. Ferruti P, Franchini J, Bencini $\mathrm{M}$ et al. Prevailingly cationic agmatine-based amphoteric polyamidoamine as a nontoxic, nonhemolytic, and "stealthlike" DNA complexing agent and transfection promoter. Biomacromolecules 8(5), 1498-1504 (2007).

43. Ferruti P, Manzoni S, Richardson SCW et al. Amphoteric linear poly(amido-amine)s as endosomolytic polymers: correlation between physicochemical and biological properties. Macromolecules 33(21), 7793-7800 (2000).

44. Richardson S, Ferruti P, Duncan R. Poly(amidoamine)s as potential endosomolytic polymers: evaluation in vitro and body distribution in normal and tumour-bearing animals. $J$. Drug Target. 6(6), 391-404 (1999).

45. Richardson SCW, Pattrick NG, Lavignac N, Ferruti P, Duncan R. Intracellular fate of bioresponsive poly(amidoamine)s in vitro and in vivo. J. Control. Release 142(1), 78-88 (2010).

46. Marchisio MA, Tongo T, Ferruti P. A selective de-heparinizer filter made of new crosslinked polymers of a poly-amido-amine structure. Experientia 1(29), 93-95 (1973).

47. Donghi D, Maggioni D, D'Alfonso G et al. Tricarbonyl-rhenium complexes of a thiolfunctionalized amphoteric poly(amidoamine). Biomacromolecules 10(12), 3273-3282 (2009).

48. Ferruti P, Casini G, Tempesti F, Barbucci R, Mastacchi R, Sarret M. Heparinizable materials (III). Heparin retention power of a poly(amido-amine) either as crosslinked resin, or surface-grafted on PVC. Biomaterials 5(4), 234-236 (1984).

49. Ferruti P, Danusso F, Franchi G, Polentarutti N, Garattini S. Effects of a series of new synthetic high polymers on cancer metastases. J. Drug Target. 16(5), 496-499 (1973).

50. Schacht E, Ferruti P, and Duncan R. Drug delivery agents incorporating mitomycin. Chem. Abstr. 595(WO 9505200), 248301a- (1994).

51. Ferruti P, Ranucci E, Trotta F et al. Synthesis, characterisation and antitumour activity of platinum(II) complexes of novel functionalised poly(amido amine)s. Macromol. Chem. Phys. 200(7), 1644-1654 (1999). 
52. Andersson L, Davies J, Duncan R et al. Poly(ethylene glycol)-poly(ester-carbonate) block copolymers carrying PEG-peptidyl-doxorubicin pendant side chains: synthesis and evaluation as anticancer conjugates. Biomacromolecules 6(2), 914-926 (2005).

53. Lavignac N, Nicholls JL, Ferruti P, Duncan R. Poly(amidoamine) conjugates containing doxorubicin bound via an acid-sensitive linker. Macromol. Biosci. 9(5), 480-487 (2009).

54. Piest M, Ankoné M, Engbersen JFJ. Carbohydrate-interactive pDNA and siRNA gene vectors based on boronic acid functionalized poly(amido amine)s. J. Control. Release 169(3), 266-275 (2013).

55. van der Aa LJ, Vader P, Storm G, Schiffelers RM, Engbersen JFJ. Intercalating quaternary nicotinamide-based poly(amido amine)s for gene delivery. J. Control. Release 195(0), 11-20 (2014).

56. Richardson SC, Pattrick NG, Man YK, Ferruti P, Duncan R. Poly(amidoamine)s as potential nonviral vectors: ability to form interpolyelectrolyte complexes and to mediate transfection in vitro. Biomacromolecules 2(3), 1023-1028 (2001).

57. Hartmann L, Häfele S, Peschka-Süss R, Antonietti M, Börner HG. Tailor-made poly(amidoamine)s for controlled complexation and condensation of DNA. Chemistry 14(7), 2025-2033 (2008).

58. Lin C, Zhong Z, Lok MC et al. Linear poly(amido amine)s with secondary and tertiary amino groups and variable amounts of disulfide linkages: synthesis and in vitro gene transfer properties. J. Control. Release 116(2), 130-137 (2006).

59. Martello F, Piest M, Engbersen JFJ, Ferruti P. Effects of branched or linear architecture of bioreducible poly(amido amine)s on their in vitro gene delivery properties. J. Control. Release 164(3), 372-379 (2012).

60. Wu C, Li J, Zhu Y, Chen J, Oupický D. Opposing influence of intracellular and membrane thiols on the toxicity of reducible polycations. Biomaterials 34(34), 8843-8850 (2013).

61. Cavalli R, Bisazza A, Sessa $\mathrm{R}$ et al. Amphoteric agmatine containing polyamidoamines as carriers for plasmid DNA in vitro and in vivo delivery. Biomacromolecules 11(10), 2667-2674 (2010).

62. Pattrick NG, Richardson SC, Casolaro M, Ferruti P, Duncan R. Poly(amidoamine)-mediated intracytoplasmic delivery of ricin A-chain and gelonin. J. Control. Release 77(3), 225-232 (2001).

63. Coué G, Engbersen JFJ. Bioreducible poly(amidoamine)s with charge-reversal properties for intracellular protein delivery. J. Control. Release 148(1), e9-e11 (2010).

64. Coué G, Engbersen JFJ. Functionalized linear poly(amidoamine)s are efficient vectors for intracellular protein delivery. J. Control. Release 152(1), 90-98 (2011).

65. Coué G, Freese C, Unger RE, Kirkpatrick CJ, Engbersen JFJ. Bioresponsive poly(amidoamine)s designed for intracellular protein delivery. Acta Biomater. 9(4), 60626074 (2013).

66. Ferruti P, Mauro N, Falciola L et al. Amphoteric, prevailingly cationic L-arginine polymers of poly(amidoamino acid) structure: synthesis, acid/base properties and preliminary cytocompatibility and cell-permeating characterizations. Macromol. Biosci. 14(3), 390-400 (2014).

67. Bencini M, Ranucci E, Ferruti $\mathrm{P}$ et al. Preparation and in vitro evaluation of the antiviral activity of the Acyclovir complex of a beta-cyclodextrin/poly(amidoamine) copolymer. $J$. Control. Release 126(1), 17-25 (2008).

68. Donalisio M, Ranucci E, Cagno V et al. Agmatine-containing poly(amidoamine)s as a novel class of antiviral macromolecules: structural properties and in vitro evaluation of infectivity inhibition. Antimicrob. Agents Chemother. 58(10), 6315-6319 (2014).

69. Cagno V, Donalisio M, Bugatti A et al. The agmatine-containing poly(amidoamine) polymer AGMA1 binds cell surface heparan sulfates and prevents the attachment of mucosal human papillomaviruses. Antimicrob. Agents Chemother. 59(9), 5250-5259 (2015). 
70. Urbán P, Valle-Delgado JJ, Mauro N et al. Use of poly(amidoamine) drug conjugates for the delivery of antimalarials to Plasmodium. J. Control. Release 177, 84-95 (2014).

** In this paper the potential of different PAAs as antimalarial carriers is explored and the results presented indicate that they are promising candidates for malaria therapy.

71. Pettit MW, Griffiths P, Ferruti P, Richardson SC. Poly(amidoamine) polymers: soluble linear amphiphilic drug-delivery systems for genes, proteins and oligonucleotides. Ther. Deliv. 2(7), 907-917 (2011).

72. Lavignac N, Lazenby M, Foka P et al. Synthesis and endosomolytic properties of poly(amidoamine) block copolymers. Macromol. Biosci. 4(10), 922-929 (2004).

73. Goodyer ID, Pouvelle B, Schneider TG, Trelka DP, Taraschi TF. Characterization of macromolecular transport pathways in malaria-infected erythrocytes. Mol. Biochem. Parasitol. 87(1), 13-28 (1997).

74. Kirk K. Membrane transport in the malaria-infected erythrocyte. Physiol. Rev. 81(2), 495537 (2001).

75. Delves M, Plouffe D, Scheurer C et al. The activities of current antimalarial drugs on the life cycle stages of Plasmodium: a comparative study with human and rodent parasites. PLoS Med. 9(2), e1001169-e1001169 (2012).

76. Delves MJ. Plasmodium cell biology should inform strategies used in the development of antimalarial transmission-blocking drugs. Future Med. Chem. 4(18), 2251-2263 (2012).

77. Paaijmans K, Fernàndez-Busquets X. Antimalarial drug delivery to the mosquito: an option worth exploring? Future Microbiol. 9(5), 579-582 (2014).

78. Marois E. The multifaceted mosquito anti-Plasmodium response. Curr. Opin. Microbiol. 14(4), 429-435 (2011).

79. Sinden R. A biologist's perspective on malaria vaccine development. Hum. Vaccin. 6(1), 311 (2010).

80. Alonso PL, Brown G, Arevalo-Herrera $\mathrm{M}$ et al. A research agenda to underpin malaria eradication. PLoS Med. 8(1), e1000406- (2011).

81. Silvestrini F, Bozdech Z, Lanfrancotti A et al. Genome-wide identification of genes upregulated at the onset of gametocytogenesis in Plasmodium falciparum. Mol. Biochem. Parasitol. 143(1), 100-110 (2005).

82. Bounkeua V, Li F, Vinetz JM. In vitro generation of Plasmodium falciparum ookinetes. Am. J. Trop. Med. Hyg. 83(6), 1187-1194 (2010).

83. Ghosh A, Dinglasan R, Ikadai H, Jacobs-Lorena M. An improved method for the in vitro differentiation of Plasmodium falciparum gametocytes into ookinetes. Malar. J. 9(1), 194(2010).

84. McClean CM, Alvarado HG, Neyra V, Llanos-Cuentas A, Vinetz JM. Optimized in vitro production of Plasmodium vivax ookinetes. Am. J. Trop. Med. Hyg. 83(6), 1183-1186 (2010).

85. Pfahler JM, Galinski MR, Barnwell JW, Lanzer M. Transient transfection of Plasmodium vivax blood stage parasites. Mol. Biochem. Parasitol. 149(1), 99-101 (2006).

86. Yoeli M, Upmanis RS. Plasmodium berghei ookinete formation in vitro. Exp. Parasitol. 22(1), 122-128 (1968).

87. Chaccour C, Kobylinski K, Bassat Q et al. Ivermectin to reduce malaria transmission: a research agenda for a promising new tool for elimination. Malar. J. 12(1), 153- (2013).

88. Osei-Atweneboana MY, Awadzi K, Attah SK, Boakye DA, Gyapong JO, Prichard RK. Phenotypic evidence of emerging ivermectin resistance in Onchocerca volvulus. PLoS Negl. Trop. Dis. 5(3), e998- (2011).

89. Rossignol PA, Ribeiro JMC, Spielman A. Increased intradermal probing time in sporozoiteinfected mosquitoes. Am. J. Trop. Med. Hyg. 33(1), 17-20 (1984).

90. Foster WA. Mosquito sugar feeding and reproductive energetics. Annu. Rev. Entomol. 40(1), 443-474 (1995).

* This extensive work provides a good introduction to the artificial feeding of mosquitoes that can be used for drug delivery purposes. 
91. Gu W, Müller G, Schlein Y, Novak RJ, Beier JC. Natural plant sugar sources of Anopheles mosquitoes strongly impact malaria transmission potential. PLoS ONE 6(1), e15996- (2011).

92. Beier J, Müller G, Gu W, Arheart K, Schlein Y. Attractive toxic sugar bait (ATSB) methods decimate populations of Anopheles malaria vectors in arid environments regardless of the local availability of favoured sugar-source blossoms. Malar. J. 11(1), 31- (2012).

93. Nyasembe VO, Tchouassi DP, Kirwa HK et al. Development and assessment of plant-based synthetic odor baits for surveillance and control of malaria vectors. PLOS ONE 9(2), e89818(2014).

94. Nyasembe V, Teal P, Mukabana W, Tumlinson J, Torto B. Behavioural response of the malaria vector Anopheles gambiae to host plant volatiles and synthetic blends. Parasit. Vectors 5(1), 234- (2012).

95. Kelly M, Su CY, Schaber C et al. Malaria parasites produce volatile mosquito attractants. mBio 6(2):e00235-15. doi:10.1128/(2) (2015).

96. Singh AD, Wong S, Ryan CP, Whyard S. Oral delivery of double-stranded RNA in larvae of the yellow fever mosquito, Aedes aegypti: implications for pest mosquito control. J. Insect Sci. 13(1), 69- (2013).

97. Mukabana W, Mweresa C, Omusula P et al. Evaluation of low density polyethylene and nylon for delivery of synthetic mosquito attractants. Parasit. Vectors 5(1), 202- (2012).

98. Mukabana W, Mweresa C, Otieno B et al. A novel synthetic odorant blend for trapping of malaria and other african mosquito species. J. Chem. Ecol. 38(3), 235-244 (2012).

99. Whyard S, Singh AD, Wong S. Ingested double-stranded RNAs can act as species-specific insecticides. Insect Biochem. Mol. Biol. 39(11), 824-832 (2009).

100. Zhang X, Zhang J, Zhu KY. Chitosan/double-stranded RNA nanoparticle-mediated RNA interference to silence chitin synthase genes through larval feeding in the African malaria mosquito (Anopheles gambiae). Insect Mol. Biol. 19(5), 683-693 (2010).

101. Blackwell A, Johnson SN. Electrophysiological investigation of larval water and potential oviposition chemo-attractants for Anopheles gambiae s.s. Ann. Trop. Med. Parasitol. 94(4), 389-398 (2000).

102. Rinker DC, Pitts RJ, Zhou X, Suh E, Rokas A, Zwiebel LJ. Blood meal-induced changes to antennal transcriptome profiles reveal shifts in odor sensitivities in Anopheles gambiae. Proc. Natl. Acad. Sci. U. S. A. 110(20), 8260-8265 (2013).

103. Lindh J, Okal M, Herrera-Varela M et al. Discovery of an oviposition attractant for gravid malaria vectors of the Anopheles gambiae species complex. Malar. J. 14(1), 119- (2015).

104. Ancsin JB, Kisilevsky R. A binding site for highly sulfated heparan sulfate is identified in the $\mathrm{N}$ terminus of the circumsporozoite protein: significance for malarial sporozoite attachment to hepatocytes. J. Biol. Chem. 279(21), 21824-21832 (2004).

105. Dinglasan RR, Alaganan A, Ghosh AK, Saito A, van Kuppevelt TH, Jacobs-Lorena M. Plasmodium falciparum ookinetes require mosquito midgut chondroitin sulfate proteoglycans for cell invasion. Proc. Natl. Acad. Sci. U. S. A. 104(40), 15882-15887 (2007).

106. Mathias DK, Pastrana-Mena R, Ranucci E et al. A small molecule glycosaminoglycan mimetic blocks Plasmodium invasion of the mosquito midgut. PLoS Pathog. 9(11), e1003757- (2013).

** In this paper it was demonstrated that synthetic polymers can inhibit Plasmodium transition in the in the midgut of Anopheles mosquitoes.

107. Li F, Templeton TJ, Popov V et al. Plasmodium ookinete-secreted proteins secreted through a common micronemal pathway are targets of blocking malaria transmission. J. Biol. Chem. 279(25), 26635-26644 (2004). 